\title{
ON THE UNSTEADY FLOW OF TWO VISCO-ELASTIC FLUIDS BETWEEN TWO INCLINED POROUS PLATES ${ }^{1}$
}

\author{
P.R. SENGUPTA \\ University of Kalyani \\ Department of Mathematics \\ Kalyani, West Bengal, INDIA \\ T.K. RAY \\ River Research Institute \\ Mohanpur, West Bengal, INDIA \\ L. DEBNATH \\ University of Central Florida \\ Department of Mathematics \\ Orlando, Florida 32816, USA
}

\begin{abstract}
This study is concerned with both hydrodynamic and hydromagnetic unsteady slow flows of two immiscible visco-elastic fluids of Rivlin-Ericksen type between two porous parallel nonconducting plates inclined at a certain angle to the horizontal. The exact solutions for the velocity fields, skin frictions, and the interface velocity distributions are found for both fluid models. Numerical results are presented in graphs. A comparison is made between the hydrodynamic and hydromagnetic velocity profiles. It is shown that the velocity is diminished due to the presence of a transverse magnetic field.
\end{abstract}

Key words: Visco-elastic hydromagnetic and hydrodynamic fluids, skin frictions and interface velocity fields.

AMS (MOS) subject classifications: $\quad 76 \mathrm{~W} 05$.

\section{INTRODUCTION}

The study of fluid flows in a porous medium plays an important role in the recovery of crude oil from the pores of reservoir rocks by displacement with immiscible water and forming polymetric adhesive joints between the solids. Various hydrodynamic and hydromagnetic flows in different fluid configurations have received considerable attention in recent years by several researchers including Kapur [1], Kapur and Sukhla [2], Bhattacharya [3], Gupta and Goyal [4], Gupta and Singh [5-6], and Sengupta and his associates [7-9]. In spite of this progress, some problems remained unsolved. Two such problems are considered in this paper.

\footnotetext{
${ }^{1}$ Received: September, 1991. Revised: January, 1992.
} 
This study is concerned with both hydrodynamic and hydromagnetic unsteady slow flows of two immiscible visco-elastic fluids of Rivlin-Ericksen type between two porous parallel nonconducting plates inclined at a certain angle to the horizontal. The exact solutions for the velocity fields, skin frictions, and the interface velocity distributions are found for both fluid models. Numerical results are presented in graphs. The hydrodynamic and hydromagnetic velocity profiles are compared. It is shown that velocity is diminished in the latter case due to the presence of a transverse magnetic field.

\section{FORMULATION OF THE PROBLEM}

We consider the unsteady flow of two incompressible, immiscible Rivlin-Ericksen fluids, each occupying a certain height between two porous parallel stationary plates inclined at an angle $\theta$ to the horizontal. We set a Cartesian coordinate system with the $x$-axis along the interface of the two fluids and parallel to the direction of the flow while the $z$-axis is chosen upward. Assuming that $u_{j}=u_{j}(x, z, t), v_{j}=0, w_{j}=0$ and $\frac{\partial}{\partial y}=0$ where $j=1,2$, the equation of continuity $\frac{\partial u_{j}}{\partial x}=0$ leads to $u_{j}=u_{j}(z, t)$.

The unsteady equation of motion for the incompressible visco-elastic fluids in a porous medium is

$$
\frac{d u_{j}}{d t}=-\frac{1}{\rho_{j}} \frac{\partial p}{\partial x}+\left(\nu_{j}+\beta_{j} \frac{d}{d t}\right) \frac{d^{2} u_{j}}{d z^{2}}-\frac{\alpha_{j}}{k_{j}} u_{j}+g \sin \theta
$$

where $\rho_{j}, \nu_{j}, \beta_{j}, k_{j}$ and $\eta_{j}$ are densities, coefficients of kinematic viscosity, kinematic viscoelasticity coefficients, permeabilities, and coefficients of viscosity of the fluids respectively, $j=1$ refers to the first fluid $(0 \leq z \leq L)$, and $j=2$ refers to the second fluid $(-L \leq z \leq 0)$.

The required boundary conditions are

$$
\left.\begin{array}{cc}
u_{1}=0 & \text { at } z=L, \\
u_{1}=u_{0} & \text { at } z=0
\end{array}\right\} \quad \text { for the first fluid }
$$

\section{SOLUTIONS OF THE HYDRODYNAMIC PROBLEM}

With the usual initial conditions, it is convenient to introduce the Laplace transform with respect to time $t$

$$
\bar{u}_{j}=\int_{0}^{\infty} e^{-s t} u_{j} d t, \operatorname{Re}(s)>0
$$


Application of this transform reduces equation (2.1) to the form

$$
\frac{d^{2} u_{j}}{d z^{2}}-\frac{R_{j}}{N_{j}}\left(u_{j}+\frac{p_{j}}{s R_{j}}\right)=0,
$$

where $R_{j}=s+\frac{\alpha_{j}}{k_{j}}, N_{j}=\nu_{j}+s \beta_{j}$ and $-p_{j}=-\frac{1}{\rho_{j}} \frac{\partial p}{\partial x}+g \sin \theta$ with $j=1,2$.

The solution of the transformed velocity $\bar{u}_{1}$ for the first fluid is

$$
\bar{u}_{1}(z, s)=\left(\frac{u_{0}}{s}+\frac{p_{1}}{s R_{1}}\right) \frac{\sinh (L-z) \sqrt{R_{1} / N_{1}}}{s R_{1} \sinh L \sqrt{R_{1} / N_{1}}}-\frac{p_{1}}{s R_{1}} .
$$

The inverse Laplace transform gives the velocity in $0 \leq z \leq L$,

$$
\begin{aligned}
& u_{1}(z, t)=u_{0} \frac{\sinh \left\{(L-z) \frac{1}{\sqrt{k_{1}}}\right\}}{\sinh \frac{L}{\sqrt{k_{1}}}}+\frac{p_{1} k_{1}}{\nu_{1}}\left\{\frac{\sinh (L-z) / \sqrt{k_{1}}}{\sinh L / \sqrt{k_{1}}}+\frac{\sinh z / \sqrt{k_{1}}}{\sinh L / \sqrt{k_{1}}}-1\right\} \\
& +\left\{2 \pi u_{0} L^{2} \sum_{n=1}^{\infty} \frac{(-1)^{n}\left(k_{1}-\beta_{1}\right) n \sin (L-z) \frac{n \pi}{L}}{\left(L^{2}+n^{2} \pi^{2} \beta_{1}\right)\left(L^{2}+n^{2} \pi^{2} k_{1}\right)}\right\} \exp \left\{-\frac{\nu_{1}\left(\pi^{2} n^{2} k_{1}+L^{2}\right)}{k_{1}\left(n^{2} \pi^{2} \beta_{1}+L^{2}\right)} \cdot t\right\} \\
& -\frac{2 p_{1} L^{2} k_{1}}{\nu_{1} \pi} \sum_{n=1}^{\infty}(-1)^{n} \frac{\left\{\sin \frac{n \pi}{L}(L-z)+\sin \frac{n \pi z}{L}\right\}}{n\left(L^{2}+n^{2} \pi^{2} k_{1}\right)} \cdot \exp \left\{-\frac{\nu_{1}\left(\pi^{2} n^{2} k_{1}+L^{2}\right)}{k_{1}\left(n^{2} \pi^{2} \beta_{1}+L^{2}\right)} \cdot t\right\} .
\end{aligned}
$$

The transformed velocity $\bar{u}_{2}$ in $-L \leq z \leq 0$ is

$$
\begin{aligned}
& \bar{u}_{2}(z, s)=\left(\frac{u_{0}}{s}+\frac{p_{2}}{s R_{2}}\right) \frac{\sinh (L+z) \sqrt{R_{2} / N_{2}}}{\sinh L \sqrt{R_{2} / N_{2}}}-\frac{p_{2}}{s R_{2}}\left\{\frac{\sinh z \sqrt{R_{2} / N_{2}}}{\sinh L \sqrt{R_{2} / N_{2}}}+1\right\} \\
& +2 \pi u_{0} L^{2}\left(k_{2}-\beta_{2}\right) \sum_{n=1}^{\infty} \frac{n(-1)^{n} \sin (L+z) \frac{n \pi}{L}}{\left(L^{2}+n^{2} \pi^{2} \beta_{2}\right)\left(L^{2}+\pi^{2} n^{2} k_{2}\right)} \exp \left\{-\frac{\nu_{2}\left(n^{2} \pi^{2} k_{2}+L^{2}\right)}{k_{2}\left(n^{2} \pi^{2} \beta^{2}+L^{2}\right)} \cdot t\right\} \\
& -\frac{2 p_{2} L^{2} k_{2}}{\nu_{2} \pi} \sum_{n=1}^{\infty}(-1)^{n} \frac{\left\{\sin _{L}^{n \pi}(L+z)-\sin \frac{n \pi z}{L}\right\}}{n\left(L^{2}+n^{2} \pi^{2} k_{2}\right)} \exp \left\{-\frac{\nu_{2}\left(n^{2} \pi^{2} k_{2}+L^{2}\right)}{k_{2}\left(n^{2} \pi^{2} k^{2}+L^{2}\right)} \cdot t\right\} .
\end{aligned}
$$

In the limit as $t \rightarrow \infty$, the exponential terms in (3.4) and (3.6) tend to zero and hence the steady state solutions are attained and are given by the first two terms in (3.4) for $u_{1}(z, t)$, and by the first two terms in (3.6) for $u_{2}(z, t)$.

Interface Velocity: The tangential stress is continuous at the interface of the liquids. Thus we get

$$
\left.\eta_{1} \frac{\partial u_{1}}{\partial z}\right|_{z=0}=\left.\eta_{2} \frac{\partial u_{2}}{\partial z}\right|_{z=0}
$$


The interface velocity can be written as

$$
u_{0}=\frac{\frac{\eta_{1} p_{1} \sqrt{k_{1}}}{\nu_{1}}\left(\operatorname{coth} L / \sqrt{k_{1}}-\operatorname{cosech} L / \sqrt{k_{1}}\right)+\frac{p_{2} \eta_{2} \sqrt{k_{2}}}{\nu_{2}}\left(\operatorname{coth} L / \sqrt{k_{2}}-\operatorname{cosech} L / \sqrt{k_{2}}\right)}{-\left(\frac{\eta_{1}}{\sqrt{k_{1}}} \operatorname{coth} L / \sqrt{k_{1}}+\frac{\eta_{2}}{\sqrt{k_{2}}} \operatorname{coth} L / \sqrt{k_{2}}\right)} .
$$

On putting $q_{j}=-\frac{p_{j}}{2 \nu_{j} u_{0}}$ in the steady-state solutions, we obtain

$$
\frac{u_{1}}{u_{0}}=\left(1-2 q_{1} k_{1}\right) \frac{\sinh (L-z) / \sqrt{k_{1}}}{\sinh L / \sqrt{k_{1}}}-2 q_{1} k_{1}\left(\frac{\sinh z / \sqrt{k_{1}}}{\sinh L / \sqrt{k_{1}}}-1\right)
$$

and

$$
\frac{u_{2}}{u_{0}}=\left(1-2 q_{2} k_{2}\right) \frac{\sinh (L+z) / \sqrt{k_{2}}}{\sinh L / \sqrt{k_{2}}}+2 q_{2} k_{2}\left(\frac{\sinh z / \sqrt{k_{2}}}{\sinh L / \sqrt{k_{2}}}+1\right)
$$

\section{SOLUTIONS OF THE HYDRODYNAMIC PROBLEM}

We consider the visco-elastic fluids electrically conducting in the presence of a constant transverse magnetic field $B_{0}$ along the $z$-axis. Thus, the equation of motion is

$$
\frac{\partial u_{j}}{\partial t}=-\frac{1}{\rho_{i}} \frac{\partial p}{\partial x}+\left(\nu_{j}+\beta \frac{\partial}{\partial t}\right) \frac{\partial^{2} u_{j}}{\partial z^{2}}-\frac{\nu_{j} u_{j}}{k_{j}}+g \sin \theta-\frac{\sigma_{j} B_{0}^{2}}{\rho_{j}} u_{j}
$$

where $\sigma_{j}$ is the electrical conductivity of the fluids. This equation is to be solved by the same initial and boundary conditions as for the hydrodynamic problem. The Laplace transformed equation of $(4.2)$ is

$$
\frac{d^{2} \bar{u}_{j}}{d z^{2}}-\frac{M_{j}}{N_{j}}\left(\bar{u}_{j}+\frac{p_{j}}{s M_{j}}\right)=0
$$

where

$$
M_{j}=s+\frac{\nu_{j}}{k_{j}}+\frac{\sigma_{j} B_{0}^{2}}{\rho_{j}} \text { and } j=1,2
$$

The solution of (4.3) with the transformed boundary condition is

$$
\bar{u}_{1}(z, s)=\left(\frac{u_{0}}{s}+\frac{p_{1}}{s M_{1}}\right) \frac{\sinh (L-z) \sqrt{M_{1} / N_{1}}}{\sinh L \sqrt{M_{1} / N_{1}}}+\frac{p_{1}}{s M_{1}} \frac{\sinh z \sqrt{M_{1} / N_{1}}}{\operatorname{sinhL} \sqrt{M_{1} / N_{1}}}-\frac{p_{1}}{s M_{1}} .
$$

The inverse transform gives

$$
u_{1}(z, t)=\left(u_{0}+\frac{p_{1}}{\left(\frac{\nu_{1}}{k_{1}}+\frac{\sigma_{1} B_{0}^{2}}{\rho_{1}}\right)}\right) \frac{\sinh (L-z) \sqrt{\frac{1}{k_{1}}+\frac{\sigma_{1} B_{0}^{2}}{\rho_{1} \nu_{1}}}}{\sinh L \sqrt{\frac{1}{k_{1}}+\frac{\sigma_{1} B_{0}^{2}}{\rho_{1}}}}+\frac{p_{1}}{\left(\frac{\nu_{1}}{k_{1}}+\frac{\sigma_{1} B_{0}^{2}}{\rho_{1}}\right)}\left(\frac{\sinh z \sqrt{\frac{1}{k_{1}}+\frac{\sigma_{1} B_{0}^{2}}{\rho_{1} \nu_{1}}}}{\sinh L \sqrt{\frac{1}{k_{1}}+\frac{\sigma_{1} B_{0}^{2}}{\rho_{1} \nu_{1}}}}-1\right)
$$




$$
\begin{gathered}
+2 \pi u_{0} \sum_{n=1}^{\infty}(-1)^{n} \frac{n L^{2}\left(k_{1} \nu_{1} \rho_{1}-\beta_{1} \rho_{1} \nu_{1}-\sigma_{1} B_{0}^{2} \beta_{1} k_{1}\right) \sin (L-z) \frac{n \pi}{L}}{\left(L^{2}+n^{2} \pi \beta_{1}\right)\left(n^{2} \pi^{2} k_{1} \nu_{1} \rho_{1}+L^{2} \rho_{1} \nu_{1}+\sigma_{1} B_{0}^{2} L^{2} k_{1}\right)} \\
\cdot \exp \left(-\frac{\nu_{1}\left(n^{2} \pi^{2} k_{1}+L^{2}\right) \rho_{1}+\sigma_{1} B_{0}^{2} L^{2} k_{1}}{k_{1} \rho_{1}\left(L^{2}+n^{2} \pi^{2} \beta_{1}\right)} \cdot t\right) \\
-\frac{2 p_{1} L^{2} k_{1}}{\pi} \sum_{n=1}^{\infty}(-1)^{n} \frac{\rho_{1}\left(\sin (L-z) \frac{n \pi}{L}+\sin \left(\frac{n \pi}{L}\right) z\right)}{n\left(N^{2} \pi^{2} k_{1} \rho_{1} \nu_{1}+L^{2} \rho_{1} \nu_{1}+\sigma_{1} B_{0}^{2} k_{1} L^{2}\right)} \\
\cdot \exp \left(-\frac{\nu_{1}\left(n^{2} \pi^{2} k_{1}+L^{2}\right) \rho_{1}+\sigma_{1} B_{0}^{2} L^{2} k_{1}}{k_{1} \rho_{1}\left(L^{2}+n^{2} \pi^{2} \beta_{1}\right)}\right)
\end{gathered}
$$

Similarly, we find

$$
\bar{u}_{2}(z, s)=\left(\frac{u_{0}}{s}+\frac{p_{2}}{s M_{2}}\right) \frac{\sinh (L+z) \sqrt{M_{2} / N_{2}}}{\sinh L \sqrt{M_{2} / N_{2}}}-\frac{p_{2}}{s M_{2}}\left(\frac{\sinh z \sqrt{M_{2} / N_{2}}}{\sinh L \sqrt{M_{2} / N_{2}}}+1\right) .
$$

The inverse Laplace transform gives

$$
\begin{aligned}
& u_{2}(z, t)=\left(u_{0}+\frac{p_{2}}{\frac{\nu_{2}}{k_{2}}+\frac{\sigma_{2}^{2} B_{0}^{2}}{\rho_{2}}}\right) \frac{\sinh (L+z) \sqrt{\frac{1}{k_{2}}+\frac{\sigma_{2} B_{0}^{2}}{\rho_{2} \nu_{2}}}}{\sinh L \sqrt{\frac{1}{k_{2}}+\frac{\sigma_{2} B_{0}^{2}}{\rho_{2} \nu_{2}}}}-\frac{p_{2}}{\left(\frac{\nu_{2}}{k_{2}}+\frac{\sigma_{2} B_{0}^{2}}{\rho_{2}}\right)}\left(\frac{\sinh z \sqrt{\frac{1}{k_{2}}+\frac{\sigma_{2} B_{0}^{2}}{\rho_{2} \nu_{2}}}}{\sinh L \sqrt{\frac{1}{k_{2}}+\frac{\sigma_{2} B_{0}^{2}}{\rho_{2} \nu_{2}}}-1}\right) \\
& +2 \pi u_{0} L^{2} \sum_{n=1}^{\infty} \frac{n\left(k_{2} \nu_{2} \rho_{2}-\beta_{2} \nu_{2} \rho_{2}-\sigma_{2} B_{0}^{2} \beta_{2} k_{2}\right)\left(\sin (L+z) \frac{n \pi}{L}\right) \cdot(-1)^{n}}{\left(L^{2}+n^{2} \pi^{2} \beta_{2}\right)\left(N^{2} \pi^{2} k_{2} \rho_{2} \nu_{2}+L^{2} \rho_{2} \nu_{2}+L^{2} \rho_{2} \nu_{2}+\sigma^{2} B_{0}^{2} k_{2} L^{2}\right)} \cdot \\
& \cdot \exp \left(-\frac{\nu_{2}\left(n^{2} \pi^{2} k_{2}+L^{2}\right) \rho_{2}+\sigma_{2} B_{0}^{2} L^{2} k_{2}}{\left(L^{2}+n^{2} \pi^{2} \beta_{2}\right) k_{2} \rho_{2}} \cdot t\right) \\
& -\frac{2 p_{2} L^{2} k_{2}}{\pi} \sum_{n=1}^{\infty}(-1)^{n} \frac{\rho_{2}\left(\sin \frac{n \pi}{L}(L+z)-\sin \frac{n \pi z}{L}\right)}{n\left(n^{2} \pi^{2} k_{2} \rho_{2} \nu_{2}+L^{2} \rho_{2} \nu_{2}+\sigma B_{0}^{2} k_{2} L^{2}\right)} . \\
& \cdot \exp \left(-\frac{\nu_{2}\left(n^{2} \pi^{2} k_{2}+L^{2}\right) \rho_{2}+\sigma_{2} B_{0}^{2} L^{2} k_{2}}{\left(L^{2}+n^{2} \pi^{2} \beta_{2}\right) k_{2} \rho_{2}} \cdot t\right)
\end{aligned}
$$

In the limit as $t \rightarrow \infty$, the transient terms involved in (4.6) and (4.8) decay and the steady state solutions are attained and given by the first two terms in each of the results (4.6) and (4.8). These steady state solutions can be written as

$$
u_{1}(z, t)=\left(u_{0}+\frac{p_{1}}{\nu_{1} T_{1}}\right) \frac{\sinh (L-z) \sqrt{T_{1}}}{\sinh L \sqrt{T_{1}}}+\frac{p_{1}}{\nu_{1} T_{1}}\left(\frac{\sinh z \sqrt{T_{1}}}{\sinh L \sqrt{T_{1}}}-1\right)
$$


and

where

$$
u_{2}(z, t)=\left(u_{0}+\frac{p_{2}}{\nu_{2} T_{2}}\right) \frac{\sinh (L+z) \sqrt{T_{2}}}{\sinh L \sqrt{T_{2}}}-\frac{p_{2}}{\nu_{2} T_{1}}\left(\frac{\sinh z \sqrt{T_{2}}}{\sinh L \sqrt{T_{2}}}+1\right)
$$

$$
T_{j}=\frac{1}{k_{j}}+\frac{\sigma_{j} B_{0}^{2}}{\rho_{j}{ }_{j}}=\frac{1}{k_{j}}+\frac{H_{j}^{2}}{L^{2}}
$$

and $H_{j}$ are the Hartmann numbers.

Finally, the interface velocity is

$$
u_{0}=\frac{\frac{\eta_{1} p_{1}}{\nu_{i} \sqrt{T_{1}}}\left(\operatorname{coth} L \sqrt{T_{1}}-\operatorname{cosech} L \sqrt{T_{1}}\right)+\frac{\eta_{2} p_{2}}{\nu_{2} \sqrt{T_{2}}}\left(\operatorname{coth} L \sqrt{T_{2}}-\operatorname{cosech} L \sqrt{T_{2}}\right)}{-\left(\eta_{1} \sqrt{T_{1}} \operatorname{coth} L \sqrt{T_{1}}+\eta_{2} \sqrt{T_{2}} \operatorname{coth} L \sqrt{T_{2}}\right)} .
$$

form

In terms of the notation $q_{j}=-\frac{p_{j}}{2 \nu_{j} u_{0}}$, the steady state solution can be written in the

and

$$
\frac{u_{1}}{u_{0}}=\left(1-\frac{2 q_{1}}{T_{1}}\right) \frac{\sinh (L-z) \sqrt{T_{1}}}{\sinh L \sqrt{T_{1}}}-\frac{2 q_{1}}{T_{1}}\left(\frac{\sinh z \sqrt{T_{1}}}{\sinh L \sqrt{T_{1}}}-1\right)
$$

$$
\frac{u_{2}}{u_{0}}=\left(1-\frac{2 q_{2}}{T_{2}}\right) \frac{\sinh (L+z) \sqrt{T_{2}}}{\sinh L \sqrt{T_{2}}}+\frac{2 q_{2}}{T_{2}}\left(\frac{\sinh z \sqrt{T_{2}}}{\sinh L \sqrt{T_{2}}}+1\right)
$$

\section{DISCUSSIONS AND CONCLUSIONS}

It follows from the numerical calculation that, for a conducting fluid in the presence of a transverse magnetic field, the velocity on the interface is at its maximum and then gradually decreases with continuous increase of distance in the upward or downward direction according to whether the upper or lower fluid is involved. For the upper fluid, the velocity attains the minimum value zero at the upper boundary plate, while for the lower fluid, the velocity reaches the minimum value zero at the lower boundary plate. In both cases, the velocity profile is very smooth and continuous. For nonconducting fluids (or in the absence of a magnetic fields), the maximum velocity does not occur at the interface; rather, it is attained for the upper fluid at a point a bit higher than the interface, while for the lower fluid it is attained at a point a bit lower than the interface. This shows a striking difference in the flow pattern between a conducting and a nonconducting fluid mode. However, the nature of the variation of the flow patterns of the conducting and nonconducting fluids remains very similar.

In both fluid models that $u_{1} / u_{0}$ and $u_{2} / u_{0}$ increase with the increase in $q_{1}$ and $q_{2}$ where $q_{1}$ and $q_{2}$ represents the pressure gradients. On the other hand, these velocity ratios increase 
with the increase in permeabilities $k_{1}$ and $k_{2}$ of the fluids. It is important to observe that when

$\sigma_{1} B_{0}^{2} / \rho_{1} \nu_{1}=\sigma_{2} B_{0}^{2} / \rho_{2} \nu_{2}=1$, then $\frac{u_{1}}{u_{0}}=0.68$ and when $\sigma_{1} B_{0}^{2} / \rho_{1} \nu_{1}=\sigma_{2} B_{0}^{2} / \rho_{2} \nu_{2}=0, \frac{u_{1}}{u_{0}}=0.785$ for the same value of $z / L$. This means that the velocity decreases by $13 \%$ in the presence of a transverse magnetic field. Thus the decrease of the velocity for the hydromagnetic case is worth noting.

The velocity profiles are drawn in Figure 1 and Figure 2 for the hydrodynamic and hydromagnetic fluid models.

\section{ACKNOWLEDGMENT}

This work was partially supported by the University of Central Florida. Authors express grateful thanks to the referee and the Principal Editor for several suggestions.

\section{REFERENCES}

[1] J.N. Kapur, Applied Sci. Res., Section 10B (1968), pp. 183-193.

[2] J.N. Kapur and J.B. Sukhla, ZAMM, 44 (1964), pp. 268-269.

[3] R.N. Bhattacharya, Bull. Cal. Math. Soc., 60 (1970), pp. 127-136.

[4] M.C. Gupta and C.M. Goyal, Indian J. Pure and Appl. Math., 4 (1973), pp. 1250-1260.

[5] M. Gupta, Agra Univ. Journal of Research, (1978).

[6] M. Gupta and D.P. Singh, Indian J. Theoret. Phys., 32 (1984), pp. 275-283.

[7] P.R. Sengupta and S.K. Bhattacharyaa, Rev. Roum. Sci. Tech. Mech. Appl., Tome 25 (1980), pp. 171-181.

[8] P.R. Sengupta and J. Ray Mohapatra, Rev. Roum. Sci. Tech. Mech. Appl., 16 (1971), pp. 1023-1041. 

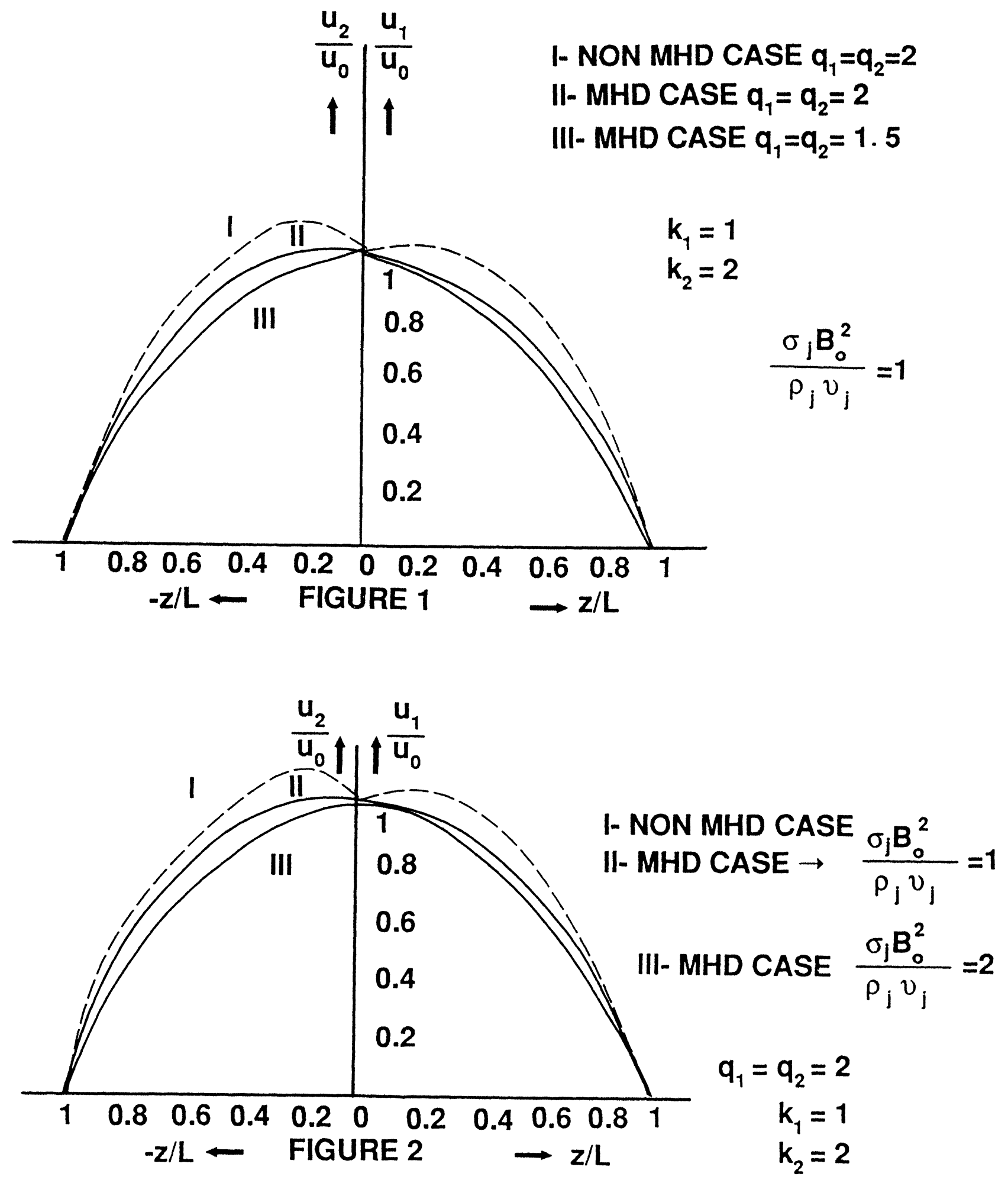


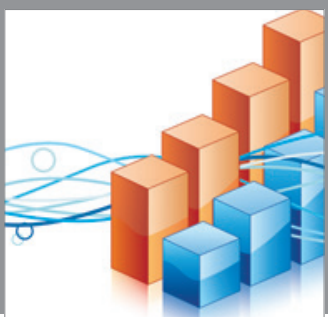

Advances in

Operations Research

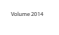

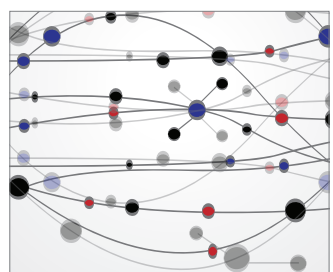

\section{The Scientific} World Journal
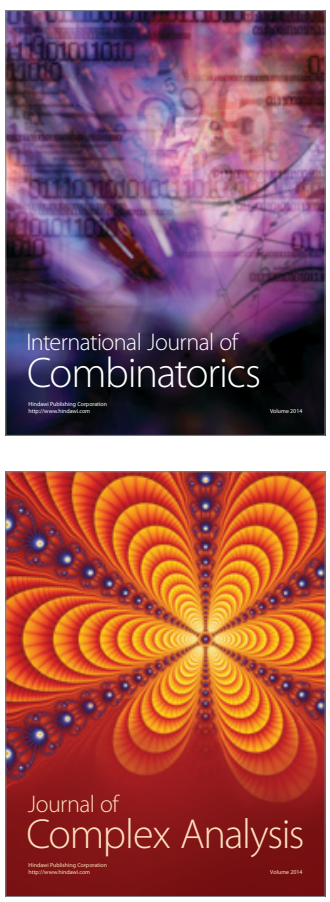

International Journal of

Mathematics and

Mathematical

Sciences
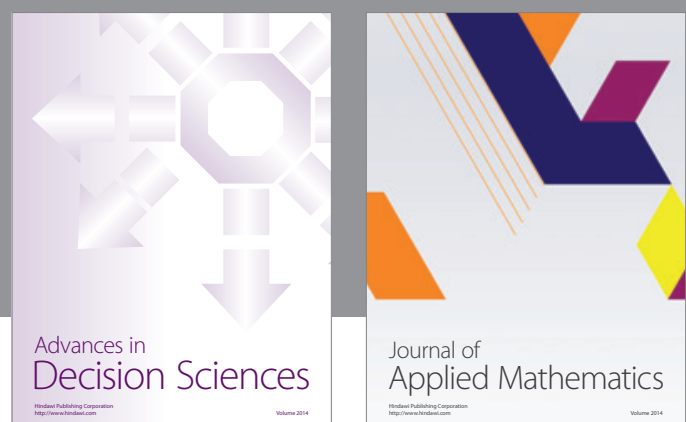

Journal of

Applied Mathematics
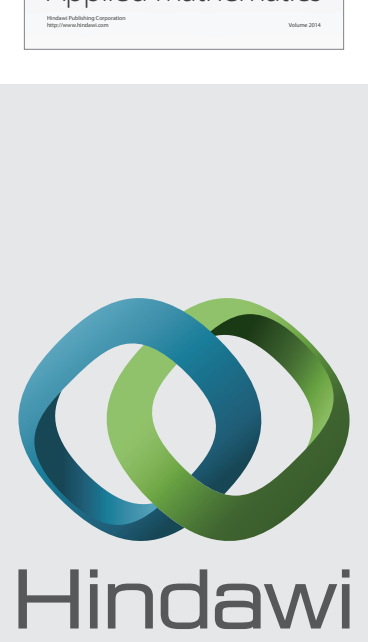

Submit your manuscripts at http://www.hindawi.com
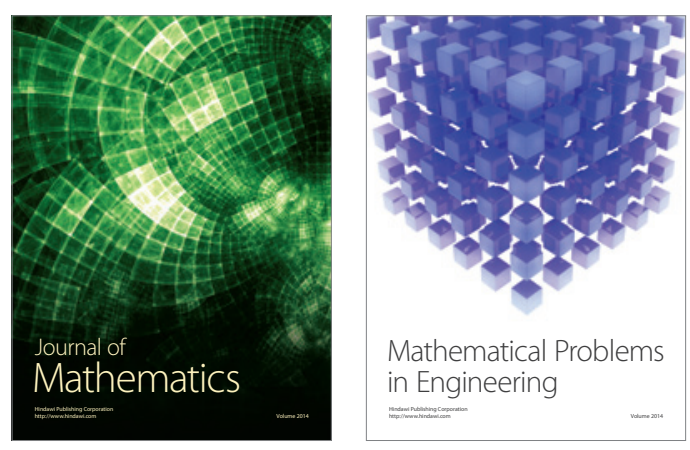

Mathematical Problems in Engineering
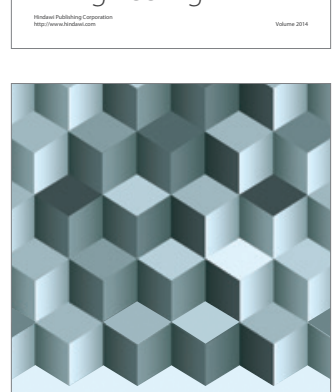

Journal of

Function Spaces
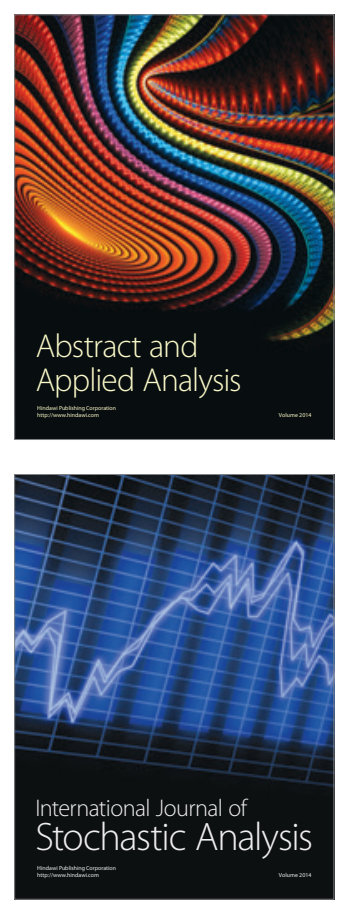

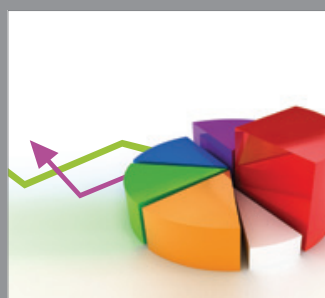

ournal of

Probability and Statistics

Promensencen
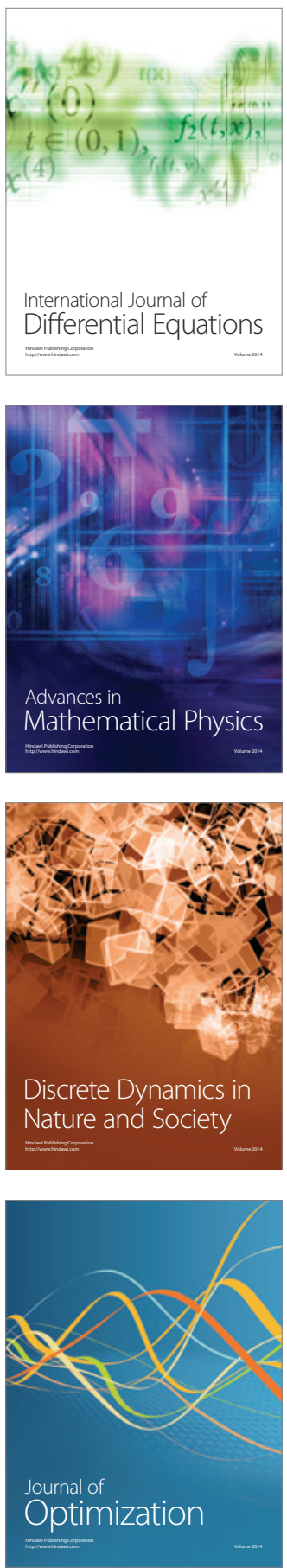